\title{
Effects of Hydrothermal Treatments on Physicochemical Properties and In Vitro Digestion of Starch
}

\author{
Ishita Chakraborty ${ }^{1} \cdot$ Indira Govindaraju ${ }^{1} \cdot$ Sintu Rongpipi ${ }^{2} \cdot$ Krishna Kishore Mahato $^{1} \cdot$ Nirmal Mazumder $^{1} \mathbb{C}$
}

Received: 5 May 2021 / Accepted: 22 July 2021 / Published online: 27 September 2021

(c) The Author(s) 2021

\begin{abstract}
Starchy food items such as rice and potato with high carbohydrate content raise blood sugar. Hence, consuming low glycaemic foods is one tool to keep diabetes under control. In this study, potato and brown rice (Njavara rice) starches were subjected to hydrothermal treatments: heat moisture treatment (HMT) and annealing (ANN) to develop starch-based food products fit for consumption by diabetic patients. The effects of hydrothermal treatments on physicochemical properties and in-vitro enzymatic digestion of starch were determined. It was observed that hydrothermal treatments decreased the swelling power (SP)\% and increased the water solubility (WS)\% of the native starches. Native potato starch (PSN) showed a high SP of $80.33 \%$, while annealed potato starch (PANN) and heat moisture treated potato starch (PHMT) showed SP reduced to $65.33 \%$ and $51.66 \%$, respectively. Similarly, the SP \% reduced from $64.33 \%$ in native brown rice (BRN) to $44.66 \%$ in annealed brown rice (BRANN) and 38.33\% in heat moisture treated brown rice (BRHMT). WS \% increased from $32.86 \%$ in PSN to $36.66 \%$ in PANN and $40.66 \%$ in PHMT. In BRN, the WS \% increased from $14.0 \%$ to $14.66 \%$ in BRANN and $18.33 \%$ in BRHMT. Amylose content increased from $13.23 \%$ and $14.56 \%$ in PSN and BRN to $16.14 \%$ in PANN $17.99 \%$ in PHMT, $17.33 \%$ in BRANN, and $18.98 \%$ in BRHMT. The PSN crystallinity index reduced from 33.49 to $30.50 \%$ in PANN and $32.60 \%$ in PHMT. At $12 \mathrm{~h}$ of enzymatic digestion, it was found that the degree of hydrolysis (DoH) of PHMT (31.66\%) and PANN (36.82\%) reduced when compared to PSN (41.09\%). Similarly, BRHMT exhibited the lowest DoH at $12 \mathrm{~h}$ compared to BRANN (29.24\%) and BRN (35.48\%). This study highlights the importance of hydrothermal treatments on starch in developing low glycaemic index commercial starch-based food products.
\end{abstract}

Keywords Starch $\cdot$ Hydrothermal treatment $\cdot$ Differential scanning calorimetry $\cdot$ X-ray diffraction

\begin{tabular}{llll}
\multicolumn{2}{l}{ Abbreviations } & AC & Amylose content \\
HMT & Heat moisture treatment & DSC & Differential Scanning Calorimetry \\
ANN & Annealing & $\mathrm{T}_{\mathrm{o}}$ & Onset temperature \\
PSN & Native potato starch & $\mathrm{T}_{\mathrm{p}}$ & Peak temperature \\
BRN & Native brown rice starch & $\mathrm{T}_{\mathrm{c}}$ & Endset temperature \\
PHMT & Heat moisture treated potato starch & $\Delta \mathrm{H}$ & Gelatinization enthalpy \\
BRHMT & Heat moisture treated brown rice starch & XRD & X-ray diffraction \\
PANN & Annealed potato starch & $\mathrm{CI}$ & Crystallinity index \\
BRANN & Annealed brown rice starch & AM & Amylose \\
SP\% & Swelling power & DoH & Degree of hydrolysis \\
WS\% & Water solubility & &
\end{tabular}

Nirmal Mazumder

nirmaluva@gmail.com

1 Department of Biophysics, Manipal School of Life Sciences, Manipal Academy of Higher Education, Manipal, Karnataka 576104, India

2 Department of Chemical Engineering, The Pennsylvania State University, University Park, PA 16802, USA

\section{Introduction}

Starch is a plant-derived polysaccharide with glucose units linked by glycosidic bonds. It further contains two polymersamylose and amylopectin. The ratio of amylose and amylopectin varies depending on its source of origin. Amylose is a chain of D-glucose monomers (linear polymer) linked by 
$\alpha-(1-4)$ while amylopectin is a branched polymer, linked by $\alpha-(1-4)$ glucan chains and $\alpha-(1-6)$ branch points [1]. The internal structure of starch granules consists of concentric rings or lamellae of alternating hard, crystalline, and soft amorphous rings. Crystalline domains or matrix of the starch granules are due to the clustered branches of amylopectin chains that are packed together, whereas the free amylose, amylose complexed with lipids, and branch points of the amylopectin are found in the amorphous region, hence, starch is said to be semi-crystalline [2]. Rice and potato are two of the most consumed sources of starch used in the food industry with total starch content ranging between 60 and $80 \%[3,4]$. However, due to their high starch content, potato and rice consumption has been frequently associated with conditions such as spiked blood sugar levels, obesity and are, hence, not prescribed for diabetics [5, 6]. Further, native starch has restricted or limited applications in the food industry due to its limited functionality, including water insolubility and susceptibility to enzymatic hydrolysis [7]. Physical modifications by hydrothermal treatments are often used to improve the functional properties such as crystallinity, gelatinization temperature, swelling power, solubility, amylose content, and enzymatic digestibility of starch. They are preferred compared to any other chemical modifications as they are less expensive, easier to perform, produce no harmful by-products, and are environmentally neutral [8]. Heat moisture treatment (HMT) and annealing (ANN) are the two most widely applied hydrothermal treatments. In HMT, starch is heated above the gelatinization temperature in the presence of inadequate moisture. On the contrary, in ANN, the starch is heated at a temperature above the glass transition temperature but below the gelatinization temperature in the presence of excess moisture. Hydrothermally treated starch is widely used in the food industry for various purposes. HMT starch is widely popular in the food industry including products of bakery and canned food items due to their increased thermal stability compared to native starch $[9,10]$. ANN is known to improve the cooking and texture qualities of rice-based cooking items such as noodles [11, 12]. Further, earlier studies demonstrated that buckwheat starch's physicochemical properties and in vitro digestibility are significantly altered on heat moisture treatment [13]. Thus, HMT and ANN are often considered to develop starch-based food items with low glycaemic index and promote the slow and steady release of glucose.

The present study was carried out to study and compare the effects of hydrothermal treatments (HMT and ANN) on physicochemical, functional properties of starch including swelling power (SP\%), water solubility (WS\%), amylose content (AC\%), crystallinity index (CI), gelatinization temperature $\left(T_{p}\right)$, and enzymatic digestibility of starch from two most conventional sources: potato, and brown rice (Njavara rice). We expect that the results of our study can be used to expand the usage of hydrothermally treated potato and rice starch as a prospective food ingredient in food industries especially for diabetic patients to reduce spiked glucose levels in the blood.

\section{Materials}

Potato starch was purchased from Hi-Media, India. The moisture content was determined to be $2 \%$. Brown rice (Njavara rice) was purchased from the local market of Kerala, India, and starch was extracted using the alkali extraction method [14]. $10 \mathrm{~g}$ of grounded rice flour was soaked in $20 \mathrm{~mL}$ of $0.1 \% \mathrm{NaOH}$ for $18 \mathrm{~h}$ at $4{ }^{\circ} \mathrm{C}$ followed by homogenization for $2 \mathrm{~min}$. The obtained slurry was centrifuged at $1400 \mathrm{rpm}$ for $10 \mathrm{~min}$. The pellet was washed four times with distilled water until a clear supernatant was obtained and the $\mathrm{pH}$ of the suspension was adjusted to 6.5. The supernatant was carefully discarded, and the pellet was then subjected to lyophilization (Power dry LL1500, Thermo Scientific, USA). The moisture content of the extracted starch was $5 \%$. The lyophilized pellet was stored at $4{ }^{\circ} \mathrm{C}$ for further experiments. $\alpha$-amylase from Bacillus subtilis $(1 \mathrm{U}=0.006 \mathrm{mg})$ was obtained from MP Biomedicals, India. 3,5-dinitro salicylic acid (DNS) was obtained from Hi-Media, India.

\section{Methods}

\section{Preparation of HMT Starch}

HMT starch was prepared by placing native potato starch (PSN) and brown rice starch (BRN) (20 g each) in sealed beakers and autoclaved at $120{ }^{\circ} \mathrm{C}$ for $60 \mathrm{~min}$. The obtained starch was dried in a hot air oven (Genaxy Scientific Pvt Ltd, India) for $48 \mathrm{~h}$. The final moisture content obtained using the oven drying method was $15 \%$. Hereafter, HMT starch from potato is referred to as PHMT and that from brown rice as BRHMT throughout the manuscript [15].

\section{Preparation of Annealed Starch}

ANN starch was prepared by heating $20 \mathrm{~g}$ of PSN and BRN in a sealed beaker along with $50 \mathrm{~mL}$ of water each at $40{ }^{\circ} \mathrm{C}$ in a water bath for $24 \mathrm{~h}$. The annealing temperature was decided based on the gelatinization temperature of the respective native starches as determined through DSC analysis as mentioned in Table 3. The starch was dried in a hot air oven (Genaxy Scientific Pvt Ltd, India) until the moisture content was approximately $10 \%$. Hence, ANN starch from potato is referred to as PANN and that from brown rice as BRANN throughout the manuscript [16]. Figure 1 depicts 
Fig. 1 Layout of physical modification of potato and brown rice starch by heat moisture treatment (HMT) and annealing (ANN). PSN, Native potato starch; BRN, Native brown rice starch; PHMT, Heat moisture treated potato starch; BRHMT, Heat moisture treated brown rice starch; PANN, Annealed potato starch; BRANN, Annealed brown rice starch

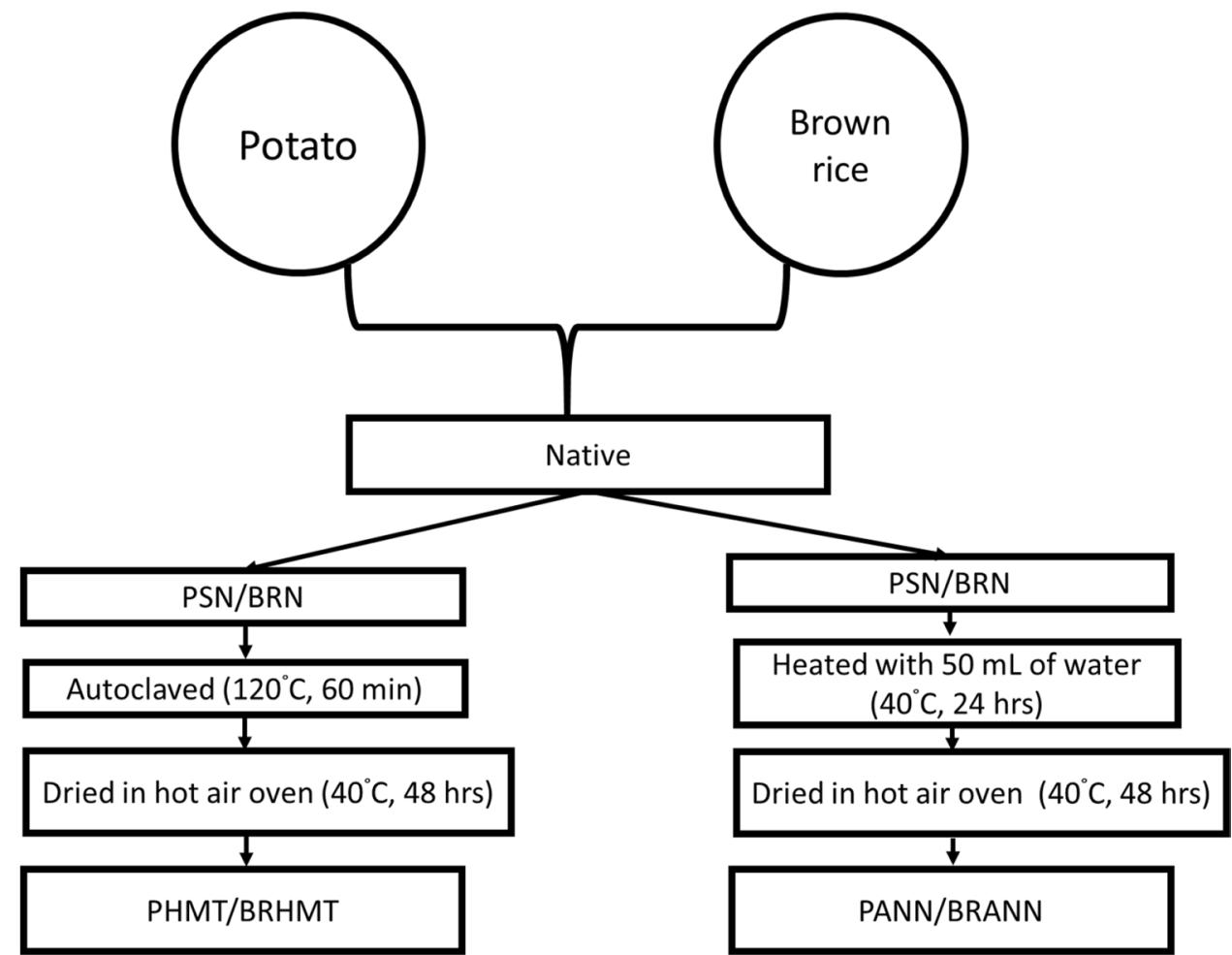

the method to obtain hydrothermally treated starch from its native form.

\section{Swelling Power (SP\%) and Water Solubility (WS\%)}

SP\% and WS\% were determined according to an earlier report with slight modifications [17]. For SP\% determination, $0.5 \mathrm{~g}$ of the native and hydrothermally treated samples (PSN, PANN, PHMT, BRN, BRANN, BRHMT) were heated in $10 \mathrm{~mL}$ distilled water in a water bath (Biobee Tech, India) at $60{ }^{\circ} \mathrm{C}$ for $30 \mathrm{~min}$ with constant stirring. This was followed by centrifugation at $1600 \mathrm{rpm}$ for $15 \mathrm{~min}$. The precipitated part was weighed and the SP\% was determined using Eq. 1. For WS\%, $0.5 \mathrm{~g}$ of the native and hydrothermally treated samples (PSN, PANN, PHMT, BRN, BRANN, BRHMT) were heated in $10 \mathrm{~mL}$ distilled water in a water bath at $60{ }^{\circ} \mathrm{C}$ for $30 \mathrm{~min}$ without any stirring. The samples were then centrifuged at $1600 \mathrm{rpm}$ for $10 \mathrm{~min}$ to obtain a pellet. The supernatant was separated, dried, and weighed in triplicate. The WS\% was determined using Eq. 2 [17]:

$$
\begin{aligned}
& \text { SP\% }=\frac{\text { Weightofsedimentalpaste }(g)}{\text { Weightofsample }(g)} * 100 \\
& W S \%=\frac{\text { Weightofsolublestarch }(g)}{\text { Weightofsample }(g)} * 2 * 100
\end{aligned}
$$

\section{Amylose Content Estimation}

The amylose content (AC\%) in both native and hydrothermally (HMT and ANN) treated starches were estimated. $100 \mathrm{mg}$ of starches were weighed and mixed with $0.36 \mathrm{~g}$ of $\mathrm{NaOH}$ in $9 \mathrm{~mL}$ of distilled water and $1 \mathrm{~mL}$ absolute ethanol and the flasks were heated in a boiling water bath for $10 \mathrm{~min}$ followed by cooling. Later, $1 \mathrm{~mL}$ acetic acid and $2 \mathrm{~mL}$ iodine solution were added. The volumes were made up to $100 \mathrm{~mL}$ and left at room temperature for $1 \mathrm{~h}$. The $200 \mu \mathrm{L}$ of the samples were loaded in 96 well plates and the optical density (OD) was then recorded at $550 \mathrm{~nm}$ in triplicates. The amylose content was calculated using Eq. 3 [14]:

$\mathrm{AC} \%=20 * 3.06^{*} \mathrm{OD}$

\section{Differential Scanning Calorimetry}

The gelatinization temperatures of native and hydrothermally (HMT and ANN) treated starches were measured using a differential scanning calorimeter, DSC (Shimadzu's DSC60, Japan). A starch sample of $3 \mathrm{mg}$ and distilled water was placed in an aluminium pan to obtain a starch to water ratio of $1: 1(\mathrm{w} / \mathrm{w})$. The pan was hermetically sealed and left for $1 \mathrm{~h}$ for equilibration. The pan was manually 
sealed using the sample-encapsulating press (Shimadzu's DSC60) and heated at specific temperatures between 30 and $150{ }^{\circ} \mathrm{C}$ with an interval of $10{ }^{\circ} \mathrm{C}$ and a flow rate of $10 \mathrm{~mL} / \mathrm{min}$. An empty aluminium pan was used as the control. The onset $\left(\mathrm{T}_{\mathrm{o}}\right)$, end set $\left(\mathrm{T}_{\mathrm{c}}\right)$, peak $\left(\mathrm{T}_{\mathrm{p}}\right)$ temperatures, and gelatinization enthalpy $(\Delta \mathrm{H}, \mathrm{J} / \mathrm{g})$ were recorded, and the curves were analyzed using the software TA-60WS and Origin 2019 software.

\section{X-ray Diffraction}

X-ray diffraction (XRD) was used to investigate the crystallinity of native and hydrothermally (HMT and ANN) treated starches. The samples were scanned through the $0.02^{\circ}$ step size, range from 3 to $60^{\circ}$ at a rate of $2^{\circ} \min ^{-1}$ using a Mini Flex benchtop XRD system (Rigaku, Japan). The results were analyzed using Origin 2019 software and the peak values were noted. The crystallinity index $(\mathrm{CI} \%)$ and amorphization degree (AM) was calculated using Eq. 4 [4]:

$C I \%=\left(\frac{A_{c r}}{A_{\text {total }}}\right) * 100$

where $\mathrm{CI}$ is the crystallinity index; $\mathrm{A}_{\mathrm{cr}}$ is the area corresponding to the crystalline phase, and $\mathrm{A}_{\text {total }}$ is a total area under the XRD pattern.

$$
\mathrm{AM}=\left(\mathrm{CI}_{0}-\mathrm{CI}_{\mathrm{t}}\right) / \mathrm{CI}_{0}
$$

where $\mathrm{AM}$ is amorphization degree; $\mathrm{CI}_{0}$ is the initial $\mathrm{CI}$ for PSN and BRN and $\mathrm{CI}_{t}$ is the CI after hydrothermal treatment (PANN, BRANN, PHMT, and BRHMT). Further, miller indices (hkl values) were determined by correlating the peak values from the literature [18].

\section{Degree of Hydrolysis}

The degree of hydrolysis (DoH\%) was calculated based on the 3,5-dinitro salicylic acid (DNS reagent) method according to reference [19] with slight modifications. $60 \mathrm{mg}$ of native and hydrothermally (HMT and ANN) treated starches were incubated with bacterial $\alpha$-amylase at a concentration of $100 \mathrm{U} / \mathrm{mL}$ in a shaker incubator for $0,1,2,3,6,9,12$, 15,18 , and $24 \mathrm{~h}$ at $40{ }^{\circ} \mathrm{C}$. The blank consisted of $60 \mathrm{mg}$ of starch sample incubated without any $\alpha$-amylase. The incubated suspensions were centrifuged at 10,000 rpm and $1 \mathrm{~mL}$ of the supernatant was incubated with $1 \mathrm{~mL}$ of DNS reagent in a boiling water bath for $10 \mathrm{~min}$. The DoH was estimated using Eq. 6 by noting the OD at $540 \mathrm{~nm}$ :

DoH $\%=\frac{(\text { ODoftestsample }- \text { ODofblank })}{(\text { ODofglucosestandard }- \text { ODofblank })} *$ concentrationofglucose

\section{Statistical Analysis}

A one-way analysis of variance (ANOVA) was used to evaluate the differences among the means of obtained values for SP\% and WS\% using Graphpad Prism 8.02. A level of $P<0.05$ was used followed by Tukey's significant difference test to compare the means of each case. Correlation coefficients were resolved using a Pearson correlation to determine a correlation between amylose content and peak gelatinization temperature $\left(T_{p}\right)$.

\section{Results}

\section{Swelling Power and Water Solubility}

PSN and BRN show a decrease in swelling power post hydrothermal treatments (HMT and ANN) compared to the native starch. While PSN shows high SP (80.33\%), PANN and PHMT show reduced SP at $65.33 \%$ and $51.66 \%$, respectively. Similarly, the SP reduces from $64.33 \%$ (BRN) to $44.66 \%$ (BRANN) and $38.33 \%$ (BRHMT). However, the WS\% of starch increases post-ANN and HMT. The WS\% value increases from $32.86 \%$ (PSN) to $36.66 \%$ (PANN) and $40.66 \%$ (PHMT). Similarly, it increases from 14\% (BRN) to $14.66 \%$ (BRANN) and $18.33 \%$ (BRHMT). The comparison of SP\% and WS\% of native and hydrothermally treated starches has been depicted in Table 1 and Fig. 2. During heating, water is first absorbed by the amorphous regions or

Table 1 Swelling power and water solubility of native and hydrothermally treated starch

\begin{tabular}{|c|c|c|c|c|c|c|}
\hline \multirow[t]{2}{*}{ Source of starch } & \multicolumn{3}{|c|}{ Swelling power $(\%)$} & \multicolumn{3}{|c|}{ Water solubility (\%) } \\
\hline & Native & ANN & HMT & Native & ANN & HMT \\
\hline Potato & $\begin{array}{l}80.33 \pm 2.51 \\
(\mathrm{PSN})\end{array}$ & $\begin{array}{l}65.33 \pm 4.72 \\
(\mathrm{PANN})\end{array}$ & $\begin{array}{l}51.66 \pm 1.15 \\
(\mathrm{PHMT})\end{array}$ & $\begin{array}{l}32.86 \pm 1.02 \\
(\mathrm{PSN})\end{array}$ & $\begin{array}{l}36.66 \pm 1.15 \\
(\mathrm{PANN})\end{array}$ & $\begin{array}{l}40.66 \pm 3.05 \\
(\mathrm{PHMT})\end{array}$ \\
\hline Brown rice (Njavara rice) & $\begin{array}{l}64.33 \pm 4.04 \\
(\mathrm{BRN})\end{array}$ & $\begin{array}{l}44.66 \pm 2.30 \\
(\mathrm{BRANN})\end{array}$ & $\begin{array}{l}38.33 \pm 1.52 \\
(\text { BRHMT) }\end{array}$ & $\begin{array}{l}14.0 \pm 2.00^{\mathrm{a}} \\
(\mathrm{BRN})\end{array}$ & $\begin{array}{l}14.66 \pm 1.15 \\
(B R A N N)\end{array}$ & $\begin{array}{l}18.33 \pm 1.52 \\
(\mathrm{BRHMT})\end{array}$ \\
\hline
\end{tabular}

PSN, Native potato starch; BRN, Native brown rice starch; PHMT, Heat moisture treated potato starch; BRHMT, Heat moisture treated brown rice starch; PANN, Annealed potato starch; BRANN, Annealed brown rice starch 

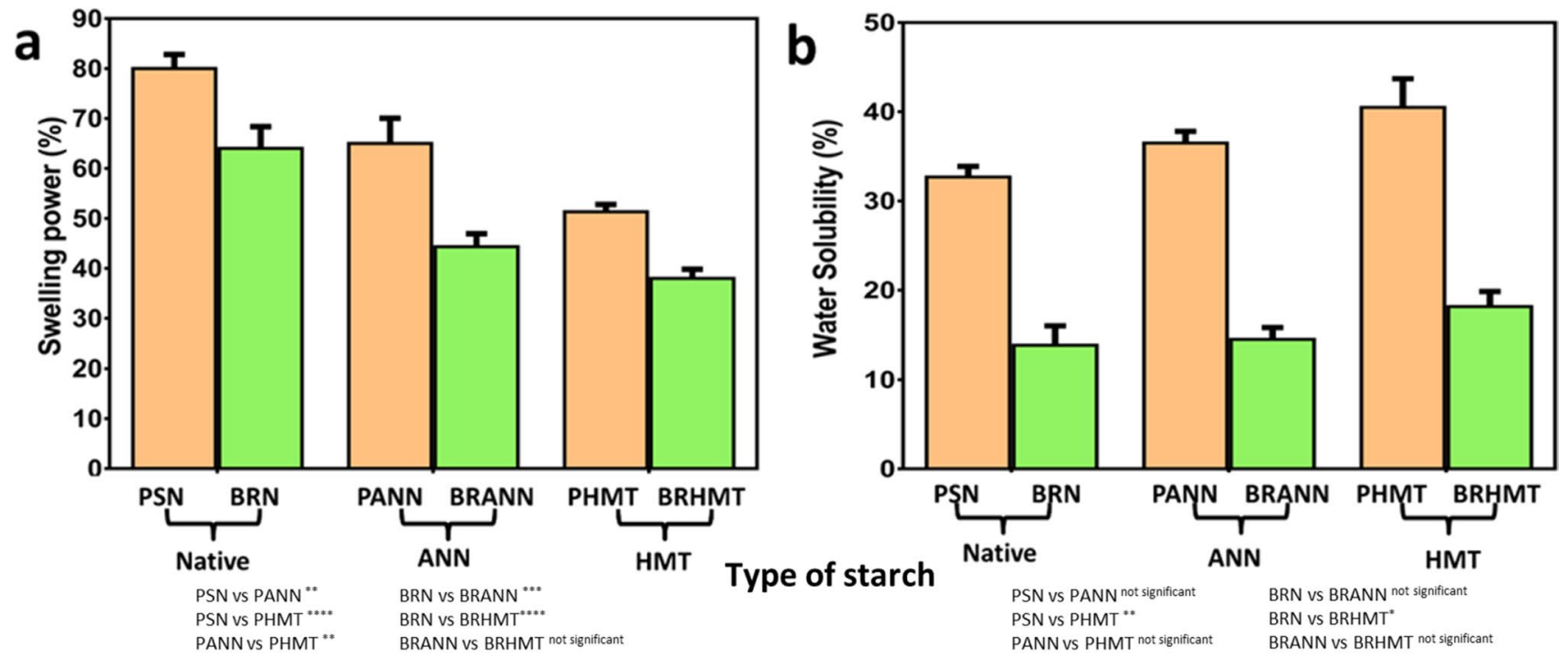

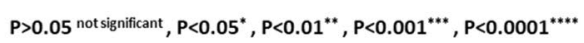

Fig. 2 Effects of hydrothermal treatments including heat moisture treatment (HMT) and annealing (ANN) on: a Swelling power (SP\%) and $\mathbf{b}$ Water solubility (WS\%) of potato and brown rice starch. Values for swelling power and water solubility for native and hydrothermally treated groups are statistically different at $* p=0.05 ; \mathrm{n}=3$. PSN,

domains of the starch granule, which leads to swelling. It is speculated that during hydrothermal treatment, rearrangement of the glucose chains takes place at the surface, preventing the entry of water into starch granules and inhibiting swelling $[9,20]$. The increase in WS\% can be attributed to the increase in $\mathrm{OH}$ group exposure to water. Hydrothermal treatments also cause physical changes like weathering of starch granules leading to better solubility in water $[15,21]$.

\section{Amylose Content Estimation}

The iodine molecule enters the $\alpha-1,4$ bonds of the amylose chain, causing a deep blue-black color, the intensity of which indicates the AC\% in starch. The AC\% is approximately similar for both PSN $(13.23 \pm 1.09 \%)$ and BRN $(14.56 \pm 2.12 \%)$. However, post hydrothermal treatment, the $\mathrm{AC} \%$ is seen to be increasing. AC\% of PANN, PHMT, BRANN, and BRHMT are $16.14 \pm 0.68 \%, 17.99 \pm 0.37 \%$, $17.33 \pm 0.30 \%$, and $18.98 \pm 1.05 \%$, respectively. Due to the treatment conditions, heat and moisture lead to degradation of the crystalline matrix and increased interaction within the amorphous matrix (amylose-amylose interactions) of the starch granule. This results in increased binding of iodine to amylose and higher color intensity during colorimetry detection. The AC \% may also vary essentially based on factors like the molecular mass of amylose and conditions of formation of amylose -iodine complex, increasing the absorbance with the increase
Native potato starch; BRN, Native brown rice starch; PHMT, Heat moisture treated potato starch; BRHMT, Heat moisture treated brown rice starch; PANN, Annealed potato starch; BRANN, Annealed brown rice starch

in these variables. HMT starch was initially treated at a higher temperature compared to ANN starch, confirming

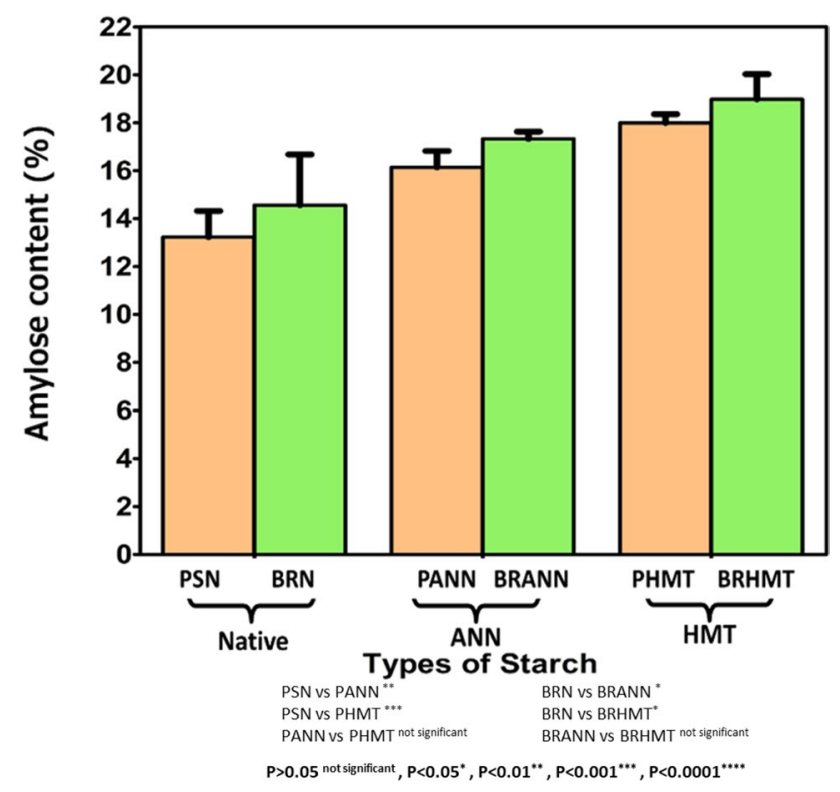

Fig. 3 Graphical representation of AC\% in native and hydrothermally treated starch samples. Values of amylose content for native and hydrothermally treated groups are statistically different at $* p=0.05$; $\mathrm{n}=3$. PSN, Native potato starch; BRN, Native brown rice starch; PHMT, Heat moisture treated potato starch; BRHMT, Heat moisture treated brown rice starch; PANN, Annealed potato starch; BRANN, Annealed brown rice starch 
Table 2 Amylose content (AC\%) of native and hydrothermally treated starch

\begin{tabular}{llll}
\hline Source of starch & AC $(\%)$ & & \\
\cline { 2 - 3 } & Native & ANN & HMT \\
\hline Potato & $13.23 \pm 1.09$ & $16.14 \pm 0.68$ & $17.99 \pm 0.37$ \\
& $(\mathrm{PSN})$ & $(\mathrm{PANN})$ & $(\mathrm{PHMT})$ \\
Brown rice (Njavara rice) & $14.56 \pm 2.12$ & $17.33 \pm 0.30$ & $18.98 \pm 1.05$ \\
& $(\mathrm{BRN})$ & $(\mathrm{BRANN})$ & $(\mathrm{BRHMT})$ \\
\hline
\end{tabular}

PSN, Native potato starch; BRN, Native brown rice starch; PHMT, Heat moisture treated potato starch; BRHMT, Heat moisture treated brown rice starch; PANN, Annealed potato starch; BRANN, Annealed brown rice starch

a direct relationship between temperature and $\mathrm{AC} \%$. Figure 3 shows a graphical representation of the AC \% in native and hydrothermally treated starch. The results showed an increase in AC \% on hydrothermal treatment of starch as depicted in Table 2.

\section{Differential Scanning Calorimetry}

Gelatinization is one of the significant functional properties that define starch quality. Starch gelatinization is the process where starch is subjected to heat and water causing irreversible swelling and bursting of the granule. PSN and BRN showed endothermic peaks typical for gelatinization of starch. PSN and BRN exhibit $\mathrm{T}_{\mathrm{p}}$ at $68.91^{\circ} \mathrm{C}$ and $57.89^{\circ} \mathrm{C}$, respectively. Figure 4 depicts the endothermic peaks of both native and hydrothermally treated starch. For both, PSN and BRN, $T_{p}$ increases post hydrothermal treatment as depicted in Table 3 and Fig. 4. PANN, PHMT, BRANN and BRHMT exhibit $\mathrm{T}_{\mathrm{p}}$ at $79.22{ }^{\circ} \mathrm{C}, 79.77{ }^{\circ} \mathrm{C}, 63.67{ }^{\circ} \mathrm{C}$, and $80.22{ }^{\circ} \mathrm{C}$, respectively. Gelatinization is controlled by two major factors-water and heat. A constraint of water penetration is caused by the formation of a new superficial layer post hydrothermal treatment. This surface transformation of starch granules by direct exposure to heat and moisture involves structural rearrangement/relocation of amylose and amylopectin (glucose) chains at the surface of the starch granules to inhibit water entry into the starch granule. The limited water condition, prevent swelling of starch granule and inhibits gelatinization [22]. Further, the higher peak temperature of starch gelatinization is accredited to the increased amylose-amylose interactions in the amorphous regions of the starch granule as mentioned earlier in Sect. 4.2. The decrease in gelatinization enthalpy $(\Delta \mathrm{H})$ has also been linked to the disruption of the crystalline domains of starch granules [23, 24]. Table 3 depicts the gelatinization
Fig. 4 DSC thermograms depicting peak gelatinisation temperatures of native and hydrothermally treated starch from $\mathbf{a}$ potato and $\mathbf{b}$ brown rice. PSN, Native potato starch; BRN, Native brown rice starch; PHMT, Heat moisture treated potato starch; BRHMT, Heat moisture treated brown rice starch; PANN, Annealed potato starch; BRANN, Annealed brown rice starch

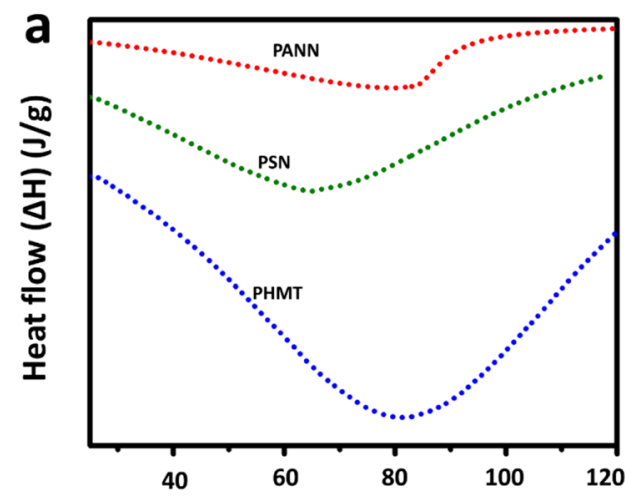

b

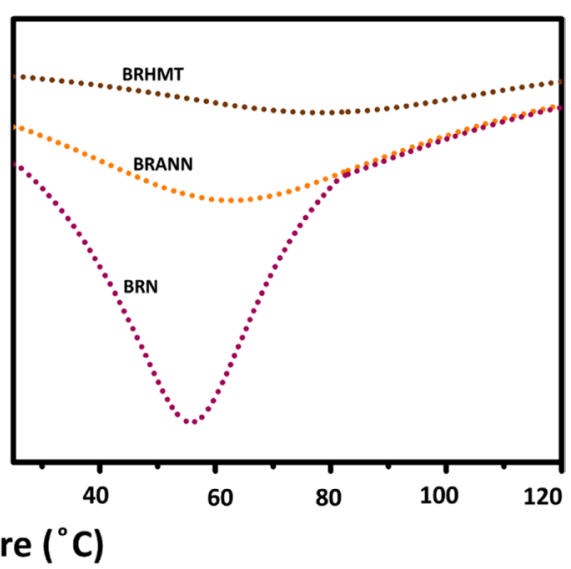

Table 3 Gelatinisation parameters of native and treated potato and brown rice starch

\begin{tabular}{lllllll}
\hline Source of starch & Type of starch & $\mathrm{T}_{\mathrm{o}}\left({ }^{\circ} \mathrm{C}\right)$ & $\mathrm{T}_{\mathrm{p}}\left({ }^{\circ} \mathrm{C}\right)$ & $\mathrm{T}_{\mathrm{c}}\left({ }^{\circ} \mathrm{C}\right)$ & Range $\left(\mathrm{T}_{\mathrm{c}}-\mathrm{T}_{\mathrm{o}}\right)$ & $\Delta \mathrm{H}(\mathrm{J} / \mathrm{g})$ \\
\hline Potato & PSN & 50.54 & 68.91 & 61.61 & 51.61 & -53.15 \\
& PANN & 62.52 & 79.22 & 87.23 & 24.71 & -50.93 \\
& PHMT & 60.42 & 79.77 & 94.06 & 33.64 & -49.37 \\
Brown rice (Njavara rice) $)$ & BRN & 51.15 & 57.89 & 87.84 & 36.3 & -99.66 \\
& BRANN & 60.05 & 63.67 & 103.27 & 42.34 & -76.30 \\
& BRHMT & 60.93 & 80.22 & 103.11 & 46.99 & -86.34 \\
\hline
\end{tabular}

PSN, Native potato starch; BRN, Native brown rice starch; PHMT, Heat moisture treated potato starch; BRHMT, Heat moisture treated brown rice starch; PANN, Annealed potato starch; BRANN, Annealed brown rice starch 
parameters of native and treated starch samples. The gelatinization range $\left(\mathrm{T}_{\mathrm{c}}-\mathrm{T}_{\mathrm{o}}\right)$ narrows post hydrothermal treatments in the case of PSN while it broadens in the case of $\mathrm{BRN}$. The gelatinization temperature range is often affected by changes within the environment, including the amount of water available and temperature [25].

\section{X-ray Diffraction}

Starch crystallinity depends on its botanical origin and the conditions used during starch modification treatment [26]. The XRD peaks and CI have been summarised in Table 4. PSN shows peaks at $2 \theta=5.5^{\circ}, 15.2^{\circ}, 17.1^{\circ}, 19.3^{\circ}, 22.2^{\circ}$, $24.3^{\circ}, 26.6^{\circ}$, exhibiting B type crystalline form. On the other hand, BRN exhibits peaks at $14.8^{\circ}, 16.7^{\circ}, 18.0^{\circ}, 19.9^{\circ}$, $23.2^{\circ}$ depicting A type crystalline form. A-type crystalline starch has densely packed amylose and amylopectin chains, whereas in B-type crystalline starch the packing is comparatively less dense, allowing more room for the incorporation of water molecules. It was observed that after hydrothermal treatment PANN and PHMT only retained peaks at approximately $2 \theta=17^{\circ}$ and $22^{\circ}$ while all other peaks were lost. However, all peaks were retained in BRANN and BRHMT compared to BRN. The XRD peaks of native and treated starch have been depicted in Fig. 5. A reduction in CI has been observed post hydrothermal treatments in both cases. The CI decreases to $32.60 \%$ (PHMT) and $30.50 \%$ (PANN) from $33.49 \%$ (PSN). Similarly, the CI decreases to $31.53 \%$ (BRHMT) and 30.79\% (BRANN) from 33.42\% (BRN). To further illustrate the effect of hydrothermal treatments on crystallinity, the 2D data of XRD diffraction patterns are shown in Figs. 5c, d, which represent a 2D stack of XRD scans with a colored ruler indicating peak intensities from strong to weak. The peak intensity decreases in post hydrothermal treatment in PHMT and PANN. However, not much change is observed in the case of BRHMT and BRANN. The reduction in CI can be attributed to the degradation of amylopectin content in the crystalline matrix and loss of long-range order in the starch granule [4, 27, 28]. In simple words, hydrothermal treatments degrade the crystalline

Table 4 XRD analysis of native and hydrothermally treated starch

\begin{tabular}{|c|c|c|c|c|c|}
\hline \multicolumn{6}{|l|}{ Potato } \\
\hline \multirow[t]{2}{*}{$2 \theta$ (degree) } & \multirow[t]{2}{*}{ Expected } & \multicolumn{3}{|c|}{ Calculated } & \multirow[t]{2}{*}{ hkl values } \\
\hline & & PSN & PANN & PHMT & \\
\hline & 5.6 & 5.5 & - & - & $(001)$ \\
\hline & 11.5 & - & - & - & (111) \\
\hline & 15.5 & 15.2 & - & - & $(140)$ \\
\hline & 17.2 & 17.1 & 17.04 & 17.1 & (131) \\
\hline & 19.7 & 19.3 & - & - & $(103)$ \\
\hline & 22.3 & 22.2 & 22.6 & 22.6 & (113) \\
\hline & 24.2 & 24.3 & - & - & (132) \\
\hline & 26.6 & 26.6 & - & - & (142) \\
\hline Crystallinity index (CI\%) & & 33.49 & 30.50 & 32.60 & \\
\hline Amorphization degree & & - & 0.089 & 0.026 & \\
\hline \multicolumn{6}{|l|}{ Brown rice (Njavara rice) } \\
\hline \multirow[t]{2}{*}{$2 \theta$ (degree) } & \multirow[t]{2}{*}{ Expected } & \multicolumn{3}{|c|}{ Calculated } & \multirow[t]{2}{*}{ hkl values } \\
\hline & & $\overline{\mathrm{BRN}}$ & BRANN & $\overline{\text { BHMT }}$ & \\
\hline & 11.6 & - & - & - & (111) \\
\hline & 15.3 & 14.8 & 15.1 & 15 & $(140)$ \\
\hline & 17.3 & 16.7 & 17.1 & 17.1 & (131) \\
\hline & 18.1 & 18.0 & 18.0 & 18.0 & $(150)$ \\
\hline & 20.1 & 19.9 & 20.1 & 20.0 & $(032)$ \\
\hline & 23.2 & 23.2 & 23.0 & 22.8 & (132) \\
\hline & 26.5 & 26.5 & 26.3 & 26.7 & $(142)$ \\
\hline Crystallinity index (CI\%) & & 33.42 & 30.79 & 31.53 & \\
\hline Amorphization degree & & & 0.078 & 0.056 & \\
\hline
\end{tabular}

PSN, Native potato starch; BRN, Native brown rice starch; PHMT, Heat moisture treated potato starch; BRHMT, Heat moisture treated brown rice starch; PANN, Annealed potato starch; BRANN, Annealed brown rice starch 
Fig. 5 XRD spectra of native and hydrothermally treated starch from a potato and $\mathbf{b}$ brown rice. Only peaks at approximately $2 \theta=17^{\circ}$ and $22^{\circ}$ were retained post hydrothermal treatment (ANN and HMT) in potato starch while most peaks are retained in case of brown rice starch. PSN, Native potato starch; BRN, Native brown rice starch; PHMT, Heat moisture treated potato starch; BRHMT, Heat moisture treated brown rice starch; PANN, Annealed potato starch; BRANN, Annealed brown rice starch
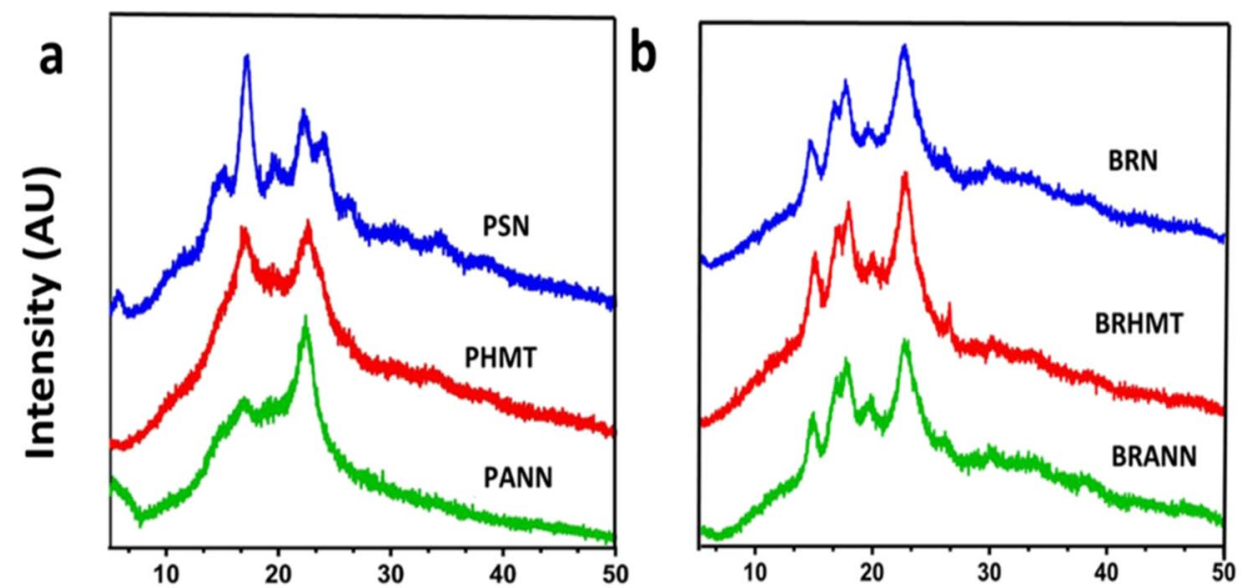

$2 \theta$ (degree)
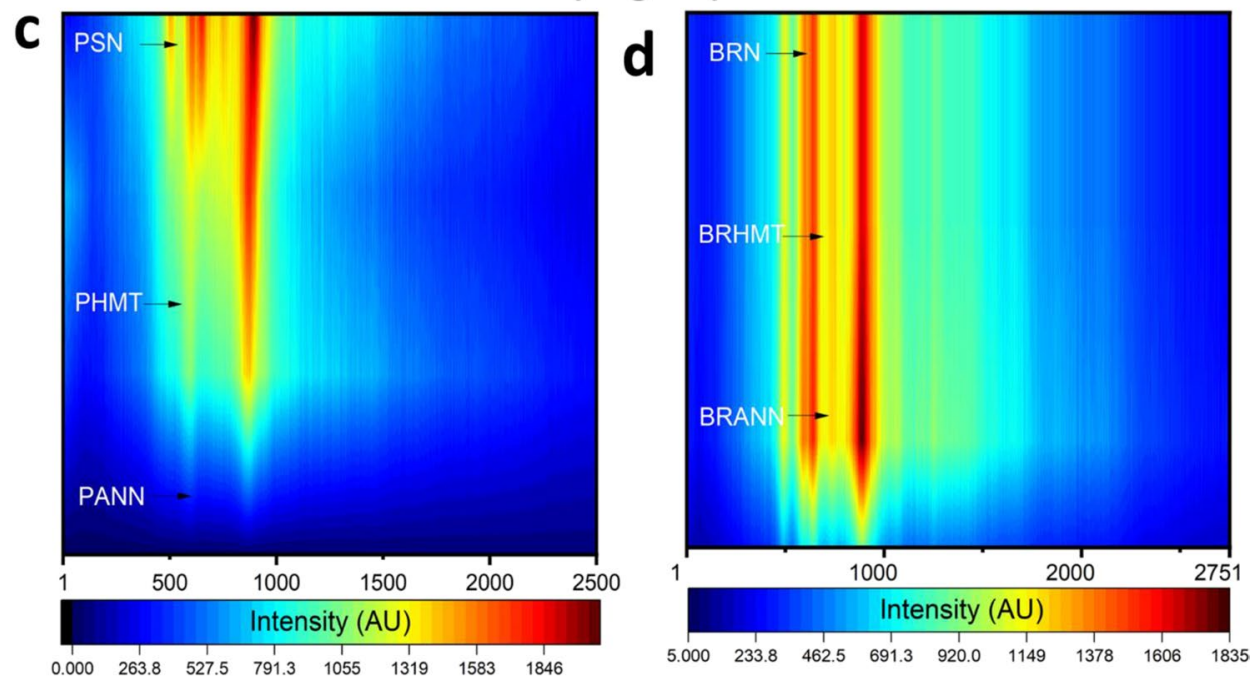

amylopectin and cause rearrangement of amylopectin and amylose chains in the amorphous matrix of the starch granule. However, the decrease in CI is more profound in B-type tuber starch compared to A-type cereal starch due to a structure of loosely packed glucose chains in the former case. These results are in accordance with Zavareze et al. (2010) where a decrease in the CI of HMT rice starch was reported [15].

\section{Degree of Starch Hydrolysis}

Alpha-amylase is an endo-acting $\alpha$-amylase that hydrolyses the $\alpha-(1-4)$ glycosidic bonds of the starch polymers internally. Enzymatic hydrolysis of starch is influenced by various factors including the ratio of amylose to amylopectin, particle size, and most importantly the crystalline structure of the granule [29]. DoH\% was calculated based on free glucose content released at different time points after hydrothermal treatment. Figure 6 and Table 5 depict the in vitro digestibility curve of native and hydrothermally treated starches digested using $\alpha$-amylase from Bacillus subtilis at a concentration of $100 \mathrm{U} /$ mL. The DoH\% was checked at 0, 1, 2, 3, 6, 9, 12, 15,18 , and $24 \mathrm{~h}$. Based on the DoH\%, PSN is more susceptible to enzymatic hydrolysis compared to BRN. This agrees with the fact that tuber starch exhibiting B-type crystalline structure with sparsely packed glucose chains facilitates easy accessibility by the hydrolyzing enzyme compared to cereal starch exhibiting A-type crystallinity with tightly packed glucose chains. The potato granules are approximately as large as $110 \mu \mathrm{m}$ whereas BRN granules are as small as $5 \mu \mathrm{m}$ concluding that larger granules are more susceptible to enzymatic hydrolysis [4, 18, 30]. DoH\% increased with prolonged digestion time $(0-24 \mathrm{~h})$ in all native and hydrothermally treated starch samples. Further, the hydrothermally treated starches are less susceptible to hydrolysis by bacterial $\alpha$-amylase compared to their native form. The reduced susceptibility of starch 


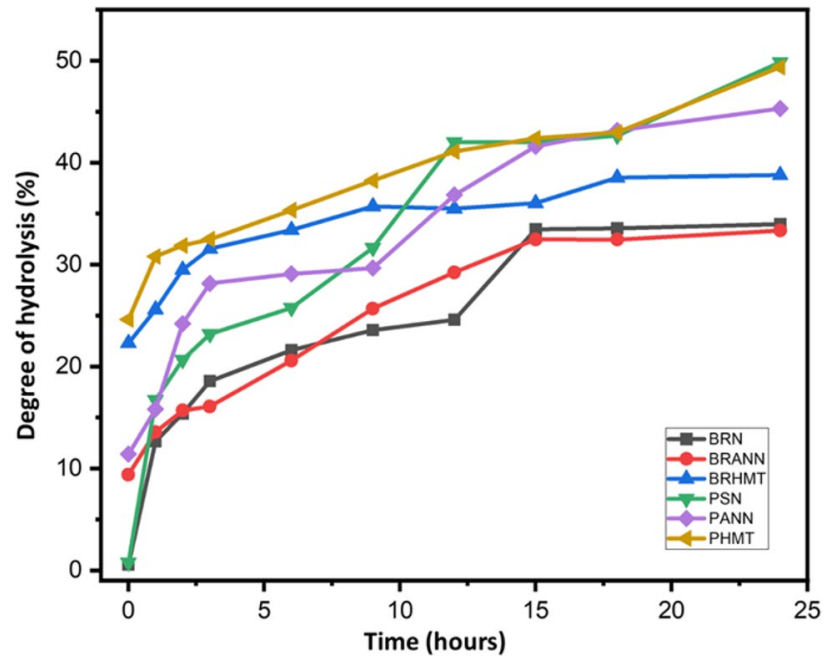

Fig. 6 Depicts the degree of hydrolysis (DoH\%) of potato and brown rice starch in with $100 \mathrm{U} / \mathrm{mL}$ of bacterial $\alpha$-amylase. PSN, Native potato starch; BRN, Native brown rice starch; PHMT, Heat moisture treated potato starch; BRHMT, Heat moisture treated brown rice starch; PANN, Annealed potato starch; BRANN, Annealed brown rice starch

to hydrolyzing enzymes is linked to the disruption of the crystalline matrix and the formation of strong interactions between strong amylose- amylose chains in the amorphous matrix [31]. Further, the decrease in enzyme susceptibility of hydrothermally treated starch is an interplay of several factors including treatment conditions (heat, pressure, and moisture), and surface rearrangement of amylose and amylopectin chains. Between HMT and ANN, starch subjected to HMT is shown to be less prone to enzymatic hydrolysis [29, 32-35].

\section{Statistical Analysis}

Statistical analysis shows that there is an overall significant difference among the means of native and hydrothermally treated starches in case of swelling power $(P<0.0001)$, as depicted in Fig. 2a. Similarly, a significant difference also exists among the means of native and hydrothermally treated starches in the case of water solubility $(P<0.05)$, as depicted in Fig. 2 b. There is also a significant difference in amylose contents before and after hydrothermal treatment for both potato and brown rice starch $(P<0.001)$, as depicted in Fig. 3. A strong positive correlation between amylose content and DSC peak temperatures $\left(\mathrm{T}_{\mathrm{p}}\right)$ was revealed ( $\mathrm{r}=0.9391 ; \mathrm{r}=0.9136$ for $P<0.05)$ for potato and rice starch, respectively.

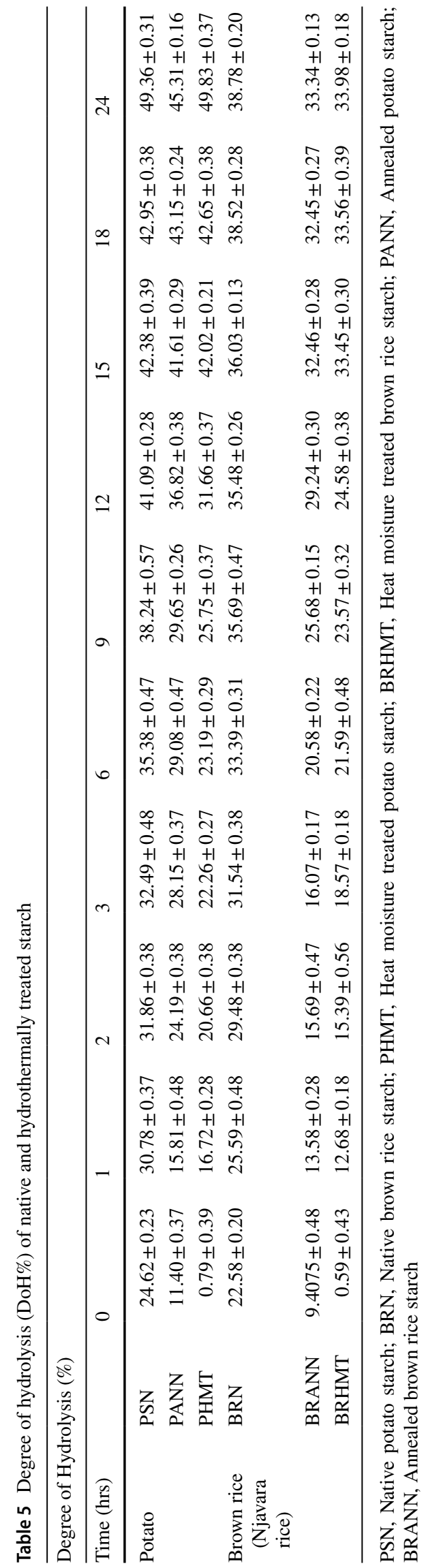




\section{Conclusion}

Starch granules are semi-crystalline, composed of amylose and amylopectin. The effects of hydrothermal treatments on physicochemical properties and in-vitro enzymatic hydrolysis on starch from potato and brown rice (Njavara rice) were studied. The hydrothermal treatments bring about a reduction in SP \% and a corresponding increase in gelatinization temperature. It was also accompanied by loss of peaks in the XRD spectra and reduction in CI [32, 36-38]. These changes in the functional properties of native starch brought about by hydrothermal treatments are essential for applications in developing starch-based food products. Hydrothermally treated starch from potato and Njavara rice of Kerala, India, can prove to be immensely effective in producing starch-based food products for diabetic patients. Hydrothermally treated starches are shown to release less glucose compared to native starch as a result of enzymatic hydrolysis, thus preventing a sudden spike in blood sugar levels. Hydrothermal treatments are also essential in several other fields, including biodegradable polymer synthesis, adhesive, and food additives.

Acknowledgements NM and KKM thank the Department of Biotechnology (DBT) and Department of Science and Technology (DST), Government of India, for the financial support (project number: BT/ PR25099/NER/95/1014/2017 and DST/INT/BLG/P-03/2019). IC, NM and KKM thank Manipal Academy of Higher Education (MAHE), Manipal and Technology Information Forecasting and Assessment Council-Centre of Relevance and Excellence (TIFAC-CORE) in Pharmacogenomics, Manipal School of Life Sciences, MAHE for providing the infrastructure and facilities. IC thank MAHE, Manipal, Karnataka, India, for the Dr. T.M.A. Pai Ph.D. fellowship.

Funding Open access funding provided by Manipal Academy of Higher Education, Manipal. We thank the Department of Biotechnology (DBT) and Department of Science and Technology (DST), Government of India, for the financial support (Project Number: BT/ PR25099/NER/95/1014/2017 and DST/INT/BLG/P-03/2019).

\section{Declarations}

Conflict of interest The authors declare no conflict of interest.

Open Access This article is licensed under a Creative Commons Attribution 4.0 International License, which permits use, sharing, adaptation, distribution and reproduction in any medium or format, as long as you give appropriate credit to the original author(s) and the source, provide a link to the Creative Commons licence, and indicate if changes were made. The images or other third party material in this article are included in the article's Creative Commons licence, unless indicated otherwise in a credit line to the material. If material is not included in the article's Creative Commons licence and your intended use is not permitted by statutory regulation or exceeds the permitted use, you will need to obtain permission directly from the copyright holder. To view a copy of this licence, visit http://creativecommons.org/licenses/by/4.0/.

\section{References}

1. B.I. Olu-Owolabi, T.A. Afolabi, K.O. Adebowale, Pasting, thermal, hydration, and functional properties of annealed and heatmoisture treated starch of sword bean (Canavalia gladiata). Int. J. Food Prop. 14(1), 157-174 (2011)

2. A. Gunaratne, X. Kong, H. Corke, Functional properties and retrogradation of heat-moisture treated wheat and potato starches in the presence of hydroxypropyl $\beta$-cyclodextrin. Starch-Stärke 62(2), 69-77 (2010)

3. L. Wang, Y.J. Wang, Rice starch isolation by neutral protease and high-intensity ultrasound. J. Cereal Sci. 39(2), 291-296 (2004)

4. K. Dome, E. Podgorbunskikh, A. Bychkov, O. Lomovsky, Changes in the crystallinity degree of starch having different types of crystal structure after mechanical pretreatment. Polymers 12(3), $641(2020)$

5. F. Baygi, M. Qorbani, M.E. Motlagh, G. Shafiee, K. Nouri, Z. Ahadi, A. Mahdavi-Gorab, R. Heshmat, R. Kelishadi, Is frequency of potato and white rice consumption associated with cardiometabolic risk factors in children and adolescents: the CASPIAN-V study. BMC Cardiovasc. Disord. 20, 1-8 (2020)

6. H. Khosravi-Boroujeni, N. Mohammadifard, N. Sarrafzadegan, F. Sajjadi, M. Maghroun, A. Khosravi, H. Alikhasi, M. Rafieian, L. Azadbakht, Potato consumption and cardiovascular disease risk factors among Iranian population. Int. J. Food Sci. Nutr. 63(8), 913-920 (2012)

7. H. O. Egharevba, Chemical properties of starch and its application in the food industry, in Chemical properties of starch (IntechOpen, 2019), pp. 63

8. S.C. Alcázar-Alay, M.A.A. Meireles, Physicochemical properties, modifications and applications of starches from different botanical sources. Food Sci. Technol. (Campinas) 35(2), 215-236 (2015)

9. K.O. Adebowalea, B.I. Olu-Owolabi, O.O. Olayinka, O.S. Lawal, Effect of heat moisture treatment and annealing on physicochemical properties of red sorghum starch. Afr. J. Biotech. 4(9), 928 933 (2005)

10. P. Khamthong, N. Lumdubwong, Effects of heat-moisture treatment on normal and waxy rice flours and production of thermoplastic flour materials. Carbohyd. Polym. 90(1), 340-347 (2012)

11. R. Hormdok, A. Noomhorm, Hydrothermal treatments of rice starch for improvement of rice noodle quality. LWT Food Sci. Technol. 40(10), 1723-1731 (2007)

12. B. Bhattacharjya, H. Dutta, K. Patwari, C.L. Mahanta, Properties of annealed jackfruit (Artocarpus heterophyllus Lam.) seed starch. Acta Aliment. 44(4), 501-510 (2015)

13. C. Goel, A.D. Semwal, A. Khan, S. Kumar, G.K. Sharma, Physical modification of starch: changes in glycemic index, starch fractions, physicochemical and functional properties of heat-moisture treated buckwheat starch. J. Food Sci. Technol. 57(8), 2941-2948 (2020)

14. P.V. Kowsik, N. Mazumder, Structural and chemical characterization of rice and potato starch granules using microscopy and spectroscopy. Microsc. Res. Tech. 81(12), 1533-1540 (2018)

15. E.D.R. Zavareze, C.R. Storck, L.A.S. de Castro, M.A. Schirmer, A.R.G. Dias, Effect of heat-moisture treatment on rice starch of varying amylose content. Food Chem. 121(2), 358-365 (2010)

16. L. Wang, C. Zhang, Z. Chen, X. Wang, K. Wang, Y. Li, J. Li, Effect of annealing on the physico-chemical properties of rice starch and the quality of rice noodles. J. Cereal Sci. 84, 125-131 (2018)

17. H. Kusumayanti, N.A. Handayani, H. Santosa, Swelling power and water solubility of cassava and sweet potatoes flour. Procedia Environ. Sci. 23, 164-167 (2015) 
18. N. Singh, J. Singh, L. Kaur, N.S. Sodhi, B.S. Gill, Morphologi$\mathrm{cal}$, thermal and rheological properties of starches from different botanical sources. Food Chem. 81(2), 219-231 (2003)

19. I. Govindaraju, S. Pallen, S. Umashankar, S.S. Mal, S. Kaniyala Melanthota, D.R. Mahato, N. Mazumder, Microscopic and spectroscopic characterization of rice and corn starch. Microsc. Res. Tech. 83(5), 490-498 (2020)

20. M.Z. Zheng, Y. Xiao, S. Yang, H.M. Liu, M.H. Liu, S. Yaqoob, J.S. Liu, Effects of heat-moisture, autoclaving, and microwave treatments on physicochemical properties of proso millet starch. Food Sci. Nutr. 8(2), 735-743 (2020)

21. Z. Sui, T. Yao, Y. Zhao, X. Ye, X. Kong, L. Ai, Effects of heatmoisture treatment reaction conditions on the physicochemical and structural properties of maize starch: moisture and length of heating. Food Chem. 173, 1125-1132 (2015)

22. R. Bertrand, W. Holmes, C. Orgeron, C. McIntyre, R. Hernandez, E.D. Revellame, Rapid estimation of parameters for gelatinization of waxy corn starch. Foods 8(11), 556 (2019)

23. D. Cooke, M.J. Gidley, Loss of crystalline and molecular order during starch gelatinisation: origin of the enthalpic transition. Carbohyd. Polym. 227, 103-112 (1992)

24. P. Khunae, T. Tran, P. Sirivongpaisal, Effect of heat-moisture treatment on structural and thermal properties of rice starches differing in amylose content. Starch-Stärke 59(12), 593-599 (2007)

25. R.N. Waduge, R. Hoover, T. Vasanthan, J. Gao, J. Li, Effect of annealing on the structure and physicochemical properties of barley starches of varying amylose content. Food Res. Int. 39(1), 59-77 (2006)

26. H.Y. Song, S.Y. Lee, S.J. Choi, K.M. Kim, J.S. Kim, G.J. Han, T.W. Moon, Digestibility and physicochemical properties of granular sweet potato starch as affected by annealing. Food Sci. Biotechnol. 23(1), 23-31 (2014)

27. M.J. Missão Cordeiro, C. Martins Veloso, L. Soares Santos, R.C. Ferreira Bonomo, M. Caliari, R.D.C. Ilhéu Fontan, The impact of heat-moisture treatment on the properties of Musa paradisiaca L. Starch and optimization of process variables. Food Technol. Biotechnol. 56(4), 506-515 (2018)

28. R. Hoove, T. Vasanthan, The effect of annealing on the physicochemical properties of wheat, oat, potato and lentil starches. J. Food Biochem. 17(5), 303-325 (1993)

29. R. Hoover, The impact of heat-moisture treatment on molecular structures and properties of starches isolated from different botanical sources. Crit. Rev. Food Sci. Nutr. 50(9), 835-847 (2010)

30. T. Zhang, C.G. Oates, Relationship between $\alpha$-amylase degradation and physico-chemical properties of sweet potato starches. Food Chem. 65(2), 157-163 (1999)

31. A. Stevnebø, S. Sahlström, B. Svihus, Starch structure and degree of starch hydrolysis of small and large starch granules from barley varieties with varying amylose content. Animal Feed Sci. Technol. 130(1), 23-38 (2006)

32. H.J. Chung, R. Hoover, Q. Liu, The impact of single and dual hydrothermal modifications on the molecular structure and physicochemical properties of normal corn starch. Int. J. Biol. Macromol. 44(2), 203-210 (2009)

33. M. Kweon, L. Haynes, L. Slade, H. Levine, The effect of heat and moisture treatments on enzyme digestibility of AeWx, Aewx and aeWx corn starches. J. Therm. Anal. Calorim. 59(1), 571-586 (2000)

34. A. Gunaratne, R. Hoover, Effect of heat-moisture treatment on the structure and physicochemical properties of tuber and root starches. Carbohyd. Polym. 49(4), 425-437 (2002)

35. E. da Rosa Zavareze, A.R.G. Dias, Impact of heat-moisture treatment and annealing in starches: a review. Carbohyd. Polym. 83(2), 317-328 (2011)

36. B. Klein, V.Z. Pinto, N.L. Vanier, Z.E. da Rosa, R. Colussi, J.A. do Evangelho, L.C. Gutkoski, A.R. Dias, Effect of single and dual heat-moisture treatments on properties of rice, cassava, and pinhao starches. Carbohydr. Polymers 98(2), 1578-84 (2013)

37. E. da Rosa Zavareze, V.Z. Pinto, B. Klein, S.L.M. El Halal, M.C. Elias, C. Prentice-Hernández, A.R.G. Dias, Development of oxidised and heat-moisture treated potato starch film. Food Chem. 132(1), 344-350 (2012)

38. K.O. Falade, O.E. Ayetigbo, Effects of annealing, acid hydrolysis and citric acid modifications on physical and functional properties of starches from four yam (Dioscorea spp.) cultivars. Food Hydrocoll 43, 529-539 (2015)

Publisher's Note Springer Nature remains neutral with regard to jurisdictional claims in published maps and institutional affiliations. 